PROCEEDINGS OF THE

AMERICAN MATHEMATICAL SOCIETY

Volume 131, Number 5, Pages 1601-1606

S 0002-9939(02)06655-8

Article electronically published on September 19, 2002

\title{
ON A CHARACTERIZATION OF MEASURES OF DISPERSION
}

\author{
A. S. FAINLEIB
}

(Communicated by Claudia M. Neuhauser)

\begin{abstract}
Measures of dispersion are characterized by the set of all bounded random variables whose dispersion is minimized when taken around the origin.
\end{abstract}

\section{IntRoduction}

Let $\varphi$ be a real valued function on $\mathbf{R}, X$ a bounded random variable (b.r.v.), and $a$ a real number. The functional $E \varphi(X-a)$ may be used as a measure of dispersion of $X$ around $a$. The base of the measure is the set of all b.r.v. $X$ such that

$$
\min _{a} E \varphi(X-a)=E \varphi(X) .
$$

For example, the base of the first absolute moment $E|X-a|$ is the set of all b.r.v. with zero median; the base of the second moment $E(X-a)^{2}$ is the set of all b.r.v. with zero mean value.

In this paper, we consider a characterization of the measures of dispersion by their bases. Kagan and Shepp 2] proved that if $\varphi$ is continuous and the base of the measure $E \varphi(X-a)$ contains all b.r.v. with $E X=0$, then $\varphi(x)=\alpha x^{2}+\varphi(0)$ with some $\alpha \geq 0$, and they also obtained a multivariate version of the result.

In what follows all the functions are real valued; $f$ is a non-negative continuous function on $\mathbf{R}$ with $f(0)=0 ; B_{\varphi}$ denotes the base of the measure $E \varphi(X-a)$ (so $B_{0}$ is the set of all b.r.v.).

Theorem 1. Let $f$ satisfy the following conditions:

$$
f(x) \text { does not vanish identically on }(-\infty, 0) \text { or on }(0, \infty)
$$

and

$$
y \int_{0}^{z}\{f(x+y)-f(x)-f(y)\} d x \geq 0 \quad \text { for any } y, z \in \boldsymbol{R} .
$$

$$
\varphi \text { is continuous on } \boldsymbol{R} \text { and } B_{f} \subseteq B_{\varphi} \text {, }
$$

then

$$
\varphi(x)=\alpha f(x)+\varphi(0)
$$

with some $\alpha \geq 0$.

Received by the editors July 5, 2001 and, in revised form, December 12, 2001. 2000 Mathematics Subject Classification. Primary 60E15. 
In particular, if $f$ is convex on $\mathbf{R}$, then (2) is equivalent to $f( \pm \infty)=\infty$. Moreover, in this case, the difference $f(x+y)-f(x)$ is an increasing function of $x$ for any fixed $y>0$ (see, for example, [1, 3.18]). Therefore, (3) is fulfilled and we have the following:

Corollary. If $f$ is convex on $\boldsymbol{R}$ and $f( \pm \infty)=\infty$, then (4) implies (5).

The bases of convex measures are described in the last section. Note that convexity of $f$ on $\mathbf{R}$ is not necessary for (3). For example, the function

$$
f(x)=x^{2}\left(x^{2}-3 x+3\right)
$$

satisfies (2) and (3) but is not convex on $\mathbf{R}$.

Theorem 2. Let $f$ be absolutely continuous on each finite interval and satisfy (2). Moreover, let $g$ be defined on $\boldsymbol{R}$, bounded on each finite interval, $g(0)=0$ and $g(x)=f^{\prime}(x)$ at all the points of differentiability of $f$ (hence, almost everywhere). If $\varphi$ is continuous on $\boldsymbol{R}$ and $B_{\varphi}$ contains all $X \in B_{0}$ with $E g(X)=0$, then (5) holds.

Condition (2) is essential. The functions

$$
f(x)=(x+|x|)^{2}, g(x)=4(x+|x|) \quad \text { and } \varphi(x)=(x+|x|)^{3}
$$

satisfy all the conditions of Theorems 1 and 2 except (2). Moreover,

$$
B_{f}=B_{\varphi}=\left\{X \in B_{0}: P(X>0)=0\right\}
$$

and $E g(X)=0$ is equivalent to $X \in B_{\varphi}$. However, (5) is obviously not valid in this case.

The functions $f(x)=|x|$ and $g(x)=\operatorname{sign} x$ satisfy all the conditions of Theorem 2. It follows from $E \operatorname{sign} X=0$ that $X$ has zero median. So if $B_{\varphi}$ contains all b.r.v. with zero median, then we have (5) with $f(x)=|x|$ (this also follows from the Corollary). The result holds under more general conditions (in particular, the function $\varphi$ may be a priori discontinuous).

Theorem 3. Let $\varphi$ be a function on $\boldsymbol{R}$ bounded from either above or below on some interval and let $0<p<1$. If $B_{\varphi}$ contains all binary r.v. $X$ with $\min X \leq 0 \leq \max X$ and $P(X=\min X)=p$, then (5) holds with

$$
f(x)=|x|+(2 p-1) x .
$$

Note that in this case $B_{f}=\left\{X \in B_{0}: P(X<0) \leq p \leq P(X \leq 0)\right\}$ (so that $B_{f}$ consists of all bounded r.v. with zero quantile of order $p$ ).

\section{Proof of Theorems 1 And 2}

Let $Y_{w}$ denote an r.v. equal to $w$ with probability 1 ,

$$
M=\{x \in \mathbf{R}: f(x)>0\}
$$

and $[M]$ is the closure of $M$. Set, moreover, for $u, v \in M$ and $u<0<v$ (there exist the such $u$ and $v$ in view of (2))

$$
\lambda=\lambda(u, v)=\{v f(u)-u f(v)\}^{-1}
$$

Let $Y=Y(u, v)$ be an r.v. with the distribution function $F(x)=F(x, u, v)$ and

$$
F(x)=\left\{\begin{array}{lll}
\lambda f(v)(x-u) & \text { for } & x \in[u, 0], \\
\lambda\{f(u) x-u f(v)\} & \text { for } & x \in[0, v] .
\end{array}\right.
$$


Lemma 1. Let $f$ satisfy (2). If $B_{\varphi}$ contains $Y_{0}, Y_{w}$ for $w \notin[M]$ and $Y(u, v)$ for $u, v \in M, u<0<v$, then (5) holds with some $\alpha \geq 0$.

Proof. It follows from $Y_{w} \in B_{\varphi}$ that

$$
\varphi(w)=E \varphi\left(Y_{w}\right)=\min _{a} E \varphi\left(Y_{w}-a\right)=\min _{t} \varphi(t) .
$$

Therefore,

$$
\varphi(0)=\varphi(w)=\min _{t} \varphi(t)
$$

for all $w \notin[M]$ and we obtain (5) for all $x \notin[M]$. Now let $u, v \in M, u<0<v$. Putting for any integrable function $r$

$$
E_{r}(z)=\operatorname{Er}(Y+z)=\lambda\left\{f(v) \int_{z+u}^{z} r(x) d x+f(u) \int_{z}^{z+v} r(x) d x\right\},
$$

and taking into account that $Y(u, v) \in B_{\varphi}$, we get $E_{\varphi}^{\prime}(0)=0$, since $\varphi$ is continuous so $E_{\varphi}(z)$ is differentiable. Hence

$$
s(u)=s(v) \quad \text { for } u, v \in M, u<0<v,
$$

where

$$
s(x)=\frac{\varphi(x)-\varphi(0)}{f(x)} .
$$

It follows that $s(x)$ has the same value $\alpha$ for all $x \in M$, so we have (5) for all such $x$. Since $f$ and $\varphi$ are continuous, it implies (5) for all $x \in[M]$ and thus for all real $x$. It follows from (5) and (7) that

$$
\min _{x} \alpha f(x)=0
$$

so $\alpha \geq 0$.

To prove Theorem 1, it is enough now to show that

$$
Y_{0}, Y_{w}, Y(u, v) \in B_{f} \quad \text { for any } u, v \in M, u<0<v, \quad \text { and any } w \notin[M] .
$$

Since

$$
f(w)=f(0)=0=\min _{t} f(t),
$$

we have $Y_{0}, Y_{w} \in B_{f}$. It follows from (3) and (8) that

$$
\int_{0}^{z} \frac{f(x+u)-f(x)}{f(u)} d x \leq z \leq \int_{0}^{z} \frac{f(x+v)-f(x)}{f(v)} d x
$$

and

$$
E_{f}(z) \geq E_{f}(0) \text { for all } z \in \mathbf{R},
$$

so $Y(u, v) \in B_{f}$ for $u, v \in M, u<0<v$.

Similarly, to prove Theorem 2, it is enough to show that $E g(X)=0$ for

$$
X=Y_{0}, Y_{w}, Y(u, v), \quad \text { where } u, v \in M, u<0<v, \quad \text { and } w \notin[M] .
$$

Indeed, $E g\left(Y_{0}\right)=g(0)=0$. If $w \notin[M]$, then $f(x)=0$ in some open interval containing $w$; therefore, also in this interval, $g(x)=f^{\prime}(x)=0$, so

$$
E g\left(Y_{w}\right)=g(w)=0 .
$$


Moreover, it follows from (8) that

$$
E g\{Y(u, v)\}=E_{g}(0)=E_{f}^{\prime}(0)=0
$$

because $f$ is absolutely continuous and so $f(x)=\int_{0}^{x} g(t) d t$ (see, for example, 4 11.7]).

\section{Proof of Theorem 3}

Continuity of $\varphi$ is essential for the proof of Theorems 1 and 2. Therefore, we now use another approach.

Let $U=U_{p}(u, v)(u<v)$ be a binary r.v. defined by

$$
P(U=u)=p, \quad P(U=v)=q=1-p .
$$

Let $x>0$ and $u \in[0, x]$. Then the r.v. $U_{p}(0, x)$ and $U_{p}(-u, x-u)$ satisfy the conditions of Theorem 3. It follows from (1) and (9) that

$$
p \varphi(-u)+q \varphi(x-u) \geq p \varphi(0)+q \varphi(x)
$$

and

$$
p \varphi(0)+q \varphi(x) \geq p \varphi(-u)+q \varphi(x-u),
$$

whence

$$
p\{\varphi(-u)-\varphi(0)\}=q\{\varphi(x)-\varphi(x-u)\} .
$$

In particular, we have by setting $x=u$ that

$$
p\{\varphi(-u)-\varphi(0)\}=q\{\varphi(u)-\varphi(0)\} .
$$

It follows from (10) and (11) that

$$
\varphi(x)-\varphi(x-u)=\varphi(u)-\varphi(0) \quad \text { for } x \geq 0, u \in[0, x]
$$

and (replacing $x$ by $u+v$ )

$$
\psi(u+v)=\psi(u)+\psi(v) \quad \text { for any } u, v \geq 0,
$$

where $\psi(x)=\varphi(x)-\varphi(0)$. So both the functions $\psi$ and $-\psi$ are convex on $[0, \infty)$ [1, 3.20]. Since one of them is bounded from above on some interval, they are continuous [1, 3.18] and therefore linear [1, 3.19]. Thus $\psi(x)=\beta x$, where $\beta$ is a constant, and

$$
\psi(x)=\frac{\beta}{2 p}\{|x|+(2 p-1) x\} \quad \text { for } x \geq 0 .
$$

In view of (11), the last equality is also valid for $x<0$. Setting $\alpha=\beta / 2 p$, we obtain (5) with $f$ defined by (6). Finally, it follows from (5) and (1) for $X=U_{p}(0,1)$ that $\alpha \geq 0$.

Remark. According to the known Blumberg-Sierpinski theorem [3], every measurable convex function is continuous. So the proof shows that the condition on $\varphi$ in Theorem 3 may be replaced by measurability of $\varphi$. 


\section{Convex measures of Dispersion}

A convex measure of dispersion is a measure $E \varphi(X-a)$ generated by a convex continuous function $\varphi$. The bases of the such measures may be described as follows.

Theorem 4. If $\varphi$ is convex and continuous on $\boldsymbol{R}$, then

$$
B_{\varphi}=\left\{X \in B_{0}: E \varphi_{-}^{\prime}(X) \leq 0 \leq E \varphi_{+}^{\prime}(X)\right\},
$$

where $\varphi_{-}^{\prime}$ and $\varphi_{+}^{\prime}$ denote the left and right derivatives of $\varphi$, respectively.

In particular, if $\varphi$ is convex and differentiable on $\mathbf{R}$, then

$$
B_{\varphi}=\left\{X \in B_{0}: E \varphi^{\prime}(X)=0\right\} .
$$

Proof. The proof of Theorem 4 is based on the following lemmas.

Lemma 2. Let functions $\psi_{n}(x)(n=1,2, \ldots)$ and their variations be uniformly bounded on an interval $[a, b]$ and let

$$
\lim _{n \rightarrow \infty} \psi_{n}(x)=\psi(x) \quad \text { for each } x \in[a, b] .
$$

If $K(x)$ is a function of bounded variation on $[a, b]$, then

$$
\lim _{n \rightarrow \infty} \int_{a}^{b} \psi_{n}(x) d K(x)=\int_{a}^{b} \psi(x) d K(x) .
$$

It is enough to prove it for the cases in which $\psi(x) \equiv 0$ and $K(x)$ is either continuous or discrete on $[a, b]$. In the first case, it follows from the known Helly's theorem by integration by parts. In the second case,

$$
I_{n}=\int_{a}^{b} \psi_{n}(x) d K(x)=\sum_{m} \psi_{n}\left(x_{m}\right) h_{m},
$$

where $m=1,2, \ldots, x_{m}$ runs over all the points of discontinuity of $K(x)$ on $[a, b]$ and $h_{m}$ are the corresponding jumps, so that

$$
\sum_{m}\left|h_{m}\right|<\infty
$$

Let $A>0,\left|\psi_{n}(x)\right| \leq A$ for all $x \in[a, b], n=1,2, \ldots$, and let $\varepsilon>0$ and

$$
\sum_{m>N}\left|h_{m}\right| \leq \varepsilon / A
$$

where $N=N(\varepsilon)$. Then

$$
\left|I_{n}\right| \leq \sum_{m \leq N}\left|\psi_{n}\left(x_{m}\right) h_{m}\right|+\varepsilon
$$

whence it follows that

$$
\limsup _{n \rightarrow \infty}\left|I_{n}\right| \leq \varepsilon, \quad \text { so } \lim _{n \rightarrow \infty} I_{n}=0,
$$

because $\psi_{n}(x) \rightarrow 0$ and $\varepsilon>0$ is arbitrary. 
Lemma 3. Let the functions $\psi_{n}(x)$ increase on $\boldsymbol{R}$ and be uniformly bounded on each finite interval. If

$$
\lim _{n \rightarrow \infty} \psi_{n}(x)=\psi(x) \quad \text { for all real } x,
$$

then

$$
\lim _{n \rightarrow \infty} E \psi_{n}(X)=E \psi(X) \quad \text { for all } X \in B_{0} .
$$

It follows immediately from the previous lemma.

Lemma 4. Let $\tau(x)$ be a convex continuous function on $\boldsymbol{R}$. Then:

(i) the ratio

$$
\frac{\tau(x+h)-\tau(x)}{h} \quad(h \neq 0)
$$

is an increasing function of $x$ and $h$, bounded for bounded $x$ and $h$;

(ii) the equality

$$
\tau\left(x_{0}\right)=\min _{x} \tau(x)
$$

is equivalent to

$$
\tau_{-}^{\prime}\left(x_{0}\right) \leq 0 \leq \tau_{+}^{\prime}\left(x_{0}\right) .
$$

It follows from known properties of convex functions [1, 3.18].

To prove Theorem 4, note that the function $\mu(x)=E \varphi(X+x)$ is also convex and continuous on $\mathbf{R}$ for any fixed $X \in B_{0}$. By Lemmas 3 and 4 ,

$$
\mu_{ \pm}^{\prime}(0)=\lim _{h \rightarrow \pm 0} E \frac{\varphi(X+h)-\varphi(X)}{h}=E \varphi_{ \pm}^{\prime}(X) .
$$

By Lemma $4, X \in B_{\varphi}$ if and only if $\mu_{-}^{\prime}(0) \leq 0 \leq \mu_{+}^{\prime}(0)$. Taking (14) into account, we obtain (13).

\section{REFERENCES}

[1] G.H. Hardy, J.E. Littlewood, and G. Polya, Inequalities. University Press, Cambridge(1934).

[2] A. Kagan and L.A. Shepp, Why the variance?, Statist. Probab. Lett. 38(1998), 329-333. MR 99c:60031

[3] W. Sierpinski, Sur les fonctions convexes mesurable, Fundamenta Math. 1(1920), 125-129.

[4] E.C. Titchmarsh, The Theory of Functions. University Press, Oxford(1939).

9-4990 Ed. Montpetit, Montreal, Quebec, Canada H3W 1P9

E-mail address: a_fainleib@hotmail.com 